\title{
The Belgian Repository of Fundamental Atomic Data and Stellar Spectra (BRASS) Identifying Fruitful Methods for Producing Atomic Data
}

\author{
Mike Laverick ${ }^{1,2, *}$, Alex Lobel ${ }^{2}$, Pierre Royer ${ }^{2}$, Christophe Martayan $^{3}$, Thibault Merle ${ }^{4}$, \\ Peter A. M. van Hoof ${ }^{2}{ }^{(D}$, Mathieu Van de Swaelmen ${ }^{4}$, Marc David ${ }^{5}{ }^{\circ}$, Herman Hensberge ${ }^{2}$ \\ and Emmanuel Thienpont ${ }^{6}$ \\ 1 Instituut voor Sterrenkunde, KU Leuven, Celestijnenlaan 200D, P.O. Box 2401, 3001 Leuven, Belgium \\ 2 Royal Observatory of Belgium, Ringlaan 3, B-1180 Brussels, Belgium; Alex.Lobel@oma.be (A.L.); \\ pierre.royer@ster.kuleuven.be (P.R.); p.vanhoof@oma.be (P.A.M.v.H.); hhensberge@gmail.com (H.H.) \\ 3 European Organisation for Astronomical Research in the Southern Hemisphere, Alonso de Córdova 3107, \\ Vitacura, 19001 Casilla, Santiago de Chile, Chile; cmartaya@eso.org \\ 4 Institut d'Astronomie et d'Astrophysique, Université Libre de Bruxelles, Av. Franklin Roosevelt 50, \\ CP 226, 1050 Brussels, Belgium; tmerle@ulb.ac.be (T.M.); Mathieu.Van.der.Swaelmen@ulb.ac.be (M.V.d.S.) \\ 5 Onderzoekgroep Toegepaste Wiskunde, Universiteit Antwerpen, Middelheimlaan 1, \\ 2020 Antwerp, Belgium; marc.david@uantwerpen.be \\ 6 Vereniging voor Sterrenkunde, Kapellebaan 56, 2811 Leest, Belgium; emmanuel.thienpont@gmail.com \\ * Correspondence: mike.laverick@kuleuven.be
}

Received: 1 July 2018; Accepted: 23 July 2018; Published: 25 July 2018

\begin{abstract}
The Belgian repository of fundamental atomic data and stellar spectra (BRASS) aims to provide the largest systematic and homogeneous quality assessment to-date of input atomic data required for stellar spectral synthesis. In addition to quality-assessed atomic data, BRASS shall also provide of a range of extremely high-quality benchmark stellar spectra spanning late B-type stars to early K-type stars. In this paper, we summarise the project's progress and available results to-date. We provide a brief comparison between our results and the BRASS project's compiled and cross-matched atomic literature, with the goal of providing useful feedback to the atomic community on which methods may produce more reliable and accurate atomic data. We hope that the examples presented here stimulate further investigation by the atomic physics community.
\end{abstract}

Keywords: atomic data; stellar spectra; oscillator strengths; atomic and molecular databases

\section{Introduction}

The Belgian repository of fundamental atomic data and stellar spectra (BRASS) aims to take the first, crucial steps towards constraining and removing systematic input errors from quantitative stellar spectroscopy [1]. The BRASS project shall perform a large-scale, homogeneous quality assessment of the atomic literature data by comparing state-of-the-art theoretical spectra against extremely high-quality observed stellar spectra. These comparisons will help to simultaneously constrain rest-wavelengths and oscillator strengths for a large number of neutral and ionic atomic transitions, spanning the optical wavelength range of 4200-6800 $\AA$, which appears in the spectrum of late B-type to early K-type stars. In addition to quality-assessing atomic data, BRASS shall also provide a range of high-quality stellar spectra, of resolution $\mathrm{R} \sim 85,000$ and signal-noise ratios of 200-1000, for the public to use. Both quality-assessed atomic data and high-quality stellar spectra will be combined together in a new interactive online database, currently under development at brass.sdf.org, which shall help astronomers and atomic physicists alike to identify spectral features and which atomic data are available. 
To date, BRASS has retrieved, cleaned, and cross-matched atomic data from a number of atomic repositories (see Laverick et al. 2018a, henceforth Paper 1 [2]), and critically evaluated the literature data against the spectra of several G-type dwarf stars including the Sun (see Laverick et al. 2018b, henceforth Paper 2 [3]). Out of the 1099 investigated spectral lines, 383 were found to be reliable for spectral synthesis, and for these lines BRASS was able to evaluate the quality of available literature data, with the goal of improving input line lists for astronomers and providing valuable feedback to atomic physicists on which literature data performs better compared to stellar spectra. In this paper ,we summarise the currently available results of the BRASS project so far (Section 2), and present a brief comparison of the literature data $\log (g f)$ values compared with benchmark $\log (g f)$ values for the 383 spectral lines (Section 3). We hope that the brief investigation presented hear will encourage further, more in-depth study by atomic physicists into which data production methods lead to more accurate and reliable atomic data.

\section{Availability of Results To-Date}

\subsection{Cross-Matched Literature}

In 2016, in preparation for our quality assessment work, BRASS retrieved a "snapshot" of the available atomic literature from several atomic databases such as VALD [4], NIST [5], and providers within the VAMDC [6]. An input atomic data line list was compiled for BRASS in 2012, using older versions of VALD and NIST, and we also have access to the SpectroWeb line list, compiled in 2008. The retrieved atomic data was homogenised, cleaned, and cross-matched, as described in Paper 1, to compare the data of multiple literature occurrences of the same physical transition. The cross-match results revealed a general consensus on transition wavelengths and energy levels, but $\log (g f)$ values were shown to vary by as much as 2 dex for a number of transitions. The results of this literature cross-match are available to view at brass.sdf.org via the "line" tab. The data can be queried per species per wavelength interval, and can be presented either as a table of atomic data, or plotted as $\log (g f)_{\text {brass }}$ against $\Delta \log (g f)_{\text {brass-database }}$ for each retrieved database.

\subsection{High-Quality Stellar Spectra}

As implied by the project acronym, BRASS shall provide a significant number of high-quality stellar spectra for the community to use. The spectra are divided into two categories: benchmark spectra and reference spectra. The benchmark spectra are extremely high-quality observations of over 20 stellar objects, spanning late B-type stars to early K-type stars. These spectra were obtained through a dedicated observation program using the HERMES spectrograph mounted on the Mercator $1.2 \mathrm{~m}$ telescope, La Palma [7]. Through co-addition of multiple exposures taken throughout a single night, the merged spectra were able to reach a signal-to-noise ratio of $\mathrm{S} / \mathrm{N} \sim 800-1000$, which, when taken at the HERMES spectral resolution of $\mathrm{R} \sim 85,000$, produces exquisite spectra with measurable spectral lines down to less than $1 \%$ of the normalised continuum. These benchmark spectra are used in the BRASS project to quality-assess the atomic literature. The reference spectra are a larger set of around 100 stellar spectra that sample the stellar parameter-space more finely than the benchmark stars. The majority of the reference spectra are taken using the MERCATOR-HERMES spectrograph with a small number extracted from the UVES-POP archive [8]. As a trade-off for their increased parameter-space coverage, the reference spectra have lower signal-to-noise ratios of $\mathrm{S} / \mathrm{N} \sim 200-500$.

Substantial work is currently under way to produce and publish online the science-ready, normalised benchmark and reference spectra. As of June 2018, several benchmark spectra are available to download via the website "download spectra" tab. The benchmark spectra are available in three forms: the extremely high-quality observed spectra, and the corresponding theoretical spectra in both broadened (to the resolution of the benchmark spectra) and unbroadened format. The benchmark spectra will be released progressively, and thus far, the benchmark spectra of stars with effective temperatures of 5000-6000 K are available: Eps Eri, 70 Oph A, 70 Vir, 51 Peg, 10 Tau, and Beta Com. 


\subsection{Interactive Online Viewing}

In addition to simply providing both quality-assessed atomic data and stellar spectra, BRASS aims to combine both aspects together in an online interactive interface. The coupling of such datasets in an online format has previously been implemented by SpectroWeb, the BRASS project's spiritual predecessor, as well as a similar line identification implementation by the NIST ASD, albeit aimed at reproducing laboratory spectra rather than stellar spectra. The completed website will allow users to view the available wavelength range for any benchmark or reference spectrum, alongside interactive labels for identified spectral features, investigated spectral lines, and quality-assessed transitions, that will provide additional atomic information. Our goal is that the BRASS website becomes a quick and reliable reference tool for astronomers to use to identify transitions in their own stellar spectra without requiring lengthy data downloads or software package installation.

The online spectra interface is currently under development, but a preliminary version is available via the website "spectra" tab. As of June 2018, several benchmark spectra are available to view interactively: the seven benchmark spectra used in our recent Paper 2 line analysis, the Neckel and Labs KPNO solar spectrum [9], and Mercator-HERMES benchmark spectra (currently unavailable for download) of Arcturus, Procyon, $68 \mathrm{Tau}$, and HR 7512. An example of the spectral labelling is implemented for atomic lines that are theoretically deeper than $2 \%$ of the normalised continuum (blue labels) and for 1099 lines investigated as part of Paper 2 (red labels). It is important to emphasise that the spectra interface is still a work-in-progress and that we expect to implement a number of substantial changes in the coming year. For functionality and usage help, we refer readers to the "help" tab of the website which will provide the most up-to-date information.

\subsection{Quality-Assessed Atomic Data}

Undoubtedly, the most important aspect of the BRASS project are the quality assessments of the atomic literature data. Our recent quality assessment work and results for lines in G-type stellar spectra were presented at the EWASS 2018 special session: atomic and molecular data needs for astronomy and astrophysics, and details of our methods and results can be found in Paper 2. Almost 1100 atomic lines were investigated and 383 lines were found to be suitable for quality assessment and are thus recommended for stellar spectral synthesis work. The quality-assessment data are available in ascii-format via Paper 2 and the brass website via dedicated webpages for each of the 1099 investigated lines. Two examples can be found at the following links: one link for a quality-assessed Fe I line, and for a Fe I line that was not suitable for quality-assessment.

\section{Comparisons Between the BRASS Results and Previously Retrieved Literature}

By comparing the 383 quality assessed, best fitting $\log (g f)$ values from Paper 2 against literature $\log (g f)$ values, it is possible to gain knowledge into which atomic data measurements and calculations lead to more accurate and reliable $\log (g f)$ values. To demonstrate the how the project results can be utilised, we have briefly investigated the quality assessment results for any insights into which methodologies could potentially be exploited. Table 1 lists all the references for which we have two or more occurrences within our 383 quality-assessed lines. To quantify how well each reference set performed, we calculated a median from the absolute values of $\Delta \log (g f)_{\text {lit-best }}$ of all relevant lines the standard deviation of the $\Delta \log (g f)_{\text {lit-best }}$ values. Taking the median of the absolute values allows us to probe the general agreement between the literature dataset and the Paper 2 results, and thus we have ordered the table in ascending order with respect to this value.

We find that some of the best performing literature sets within the cross-matched data are actually sets of experimental data, producing accurate relative transition probabilities via branching fractions, that have later been rescaled using absolute oscillator strengths determined from newer, more accurate lifetime measurements. One clear example of such a correction are the oscillator strengths of Si I lines provided by Garz (1975) [10], which were later rescaled by O'Brian and Lawler (1991) [11]. Not only 
do the majority of rescaled values produce great agreement with the Paper 2 results, but they also show substantial improvements in the median offsets compared to the unscaled sources. It is also worth noting that the unscaled sources exhibited a sizeable median offset but have relatively small standard deviations, suggesting that the distribution of relative $\log (g f)$ values is accurate, and a simple rescaling is appropriate. The re-scalings were all experimentally justified, thus it is promising that the astrophysically-derived values also detected similar offsets with respect to the unscaled sources, and could potentially be used to indicate other offsets in experimental data. We do, however, emphasise that our work entirely relies on the solar abundances of Asplund et al. (2009) [12], and as such it is possible that offsets could arise from these abundance values. Careful examination of the atomic data used in the solar abundance determinations, such as those listed by Scott et al. (2015) [13], is required before our detected offsets can truly be used to rescale experimental datasets. It is also worth noting the excellent agreement between the Paper 2 results and those of Melendez and Barbuy (2009) [14]. Melendez and Barbuy combined theoretical $g f$-values, for which multiplet line ratios are often reliable, with either: (a) experimental life-time measurements when available; or, failing that, (b) line profile measurements of the Hinkle et al. (2000) KPNO solar flux spectrum [15] to derive absolute $g f$-values for a large number of Fe II lines.

A somewhat surprising result is the agreement with the astrophysically-derived $\log (g f)$ values of Kostyk (1981, 1982a, 1982b) [16-18]. Both Kostyk and Paper 2 make use of equivalent widths to derive $\log (g f)$ values, but the implementation of the methods was expected to produce significant systematic differences. The works of Kostyk only employ one benchmark spectrum, the Liege solar spectrum atlas, whereas the Paper 2 analysis employs several benchmark spectra, including the Sun. Additionally, Kostyk makes use of the Holweger-Müller solar model using a solar micro-turbulence value of $0.8 \mathrm{~km} / \mathrm{s}$, whereas BRASS adopts the ATLAS9 atmospheric models using a solar micro-turbulence value of $1.1 \mathrm{~km} / \mathrm{s}$. Finally, Kostyk (1982a) determines a solar titanium abundance of $\log \epsilon_{T i}=4.83$, whereas Paper 2 adopts the Asplund et al. (2009) solar titanium abundance of $\log \epsilon_{T i}=4.93$, leading us to expect at least a systematic offset of roughly 0.1 dex between our results and the Kostyk (1982a) values. Despite the differences in implementation, we found the two sets of results to be in very good agreement, leading us to conclude that the multiple systematic differences between the methods have effectively cancelled each other out, leading to the derivation of the same absolute $\log (g f)$ values.

A polar opposite of the previous situation, we found that the calculations of fine-structure theoretical $\log (g f)$ values performed in Paper 1, derived from the TIPbase and TOPbase $\log (g f)$ values, performed poorly relative to the best-fitting $\log (g f)$ values. This is no doubt due to the fact that the original ab-initio single-configuration calculations of TIPbase and TOPbase made no attempt to account for fine-structure effects. More recent theoretical calculations make use of the multi-configuration Hartree-Fock method (MCHF), which accounts for configuration mixing that can lead to significant revision in $\log (g f)$ values on the order of a few dex. Given our findings, we do not recommend that fine-structure atomic data produced in the simplistic manner of Paper 1 be used for detailed spectral synthesis. It is worth noting that, due to the absence of any other alternative atomic data, the Asplund et al. (2009) solar abundance determinations of magnesium, aluminium, potassium, and calcium relied upon such calculations for the species Mg I, Al I, K I, and Ca II (detailed in [13]). Fortunately, the recent work of Pehlivan Rhodin et al. (2017) has produced new experimental measurements and theoretical calculations for a number of $\mathrm{Mg}$ I lines [19]. 
Table 1. Literature sources with data for two or more of the 383 recommended atomic lines present in G-type stellar spectra. References are sorted in ascending order according to the median of the absolute $\Delta \log (g f)$ between the given literature values and the best overall atomic data values. References with lines belonging to several species are denoted with *

\begin{tabular}{|c|c|c|c|c|}
\hline Literature Source & Species & $\mathbf{N}$ & $\begin{array}{c}\text { Median } \\
|\Delta \log (g f)|\end{array}$ & $\begin{array}{r}\text { Standard } \\
\text { Deviation }\end{array}$ \\
\hline Bard et al. (1991) rescaled using Den Hartog et al. $(2014)[20,21]$ & Fe I & 3 & 0.010 & 0.014 \\
\hline Sobeck et al. (2007) [22] & Cr I & 14 & 0.020 & 0.055 \\
\hline Kostyk (1982a) [17] & Ti I & 7 & 0.020 & 0.018 \\
\hline Garz (1973) rescaled using O'Brian and Lawler $(1991)[10,11]$ & Si I & 7 & 0.020 & 0.033 \\
\hline Blackwell et al. (1986) rescaled using Grevesse et al. (1989) [23,24] & Ti I & 3 & 0.020 & 0.037 \\
\hline Kostyk (1982b) [18] & $\mathrm{Ni}$ I & 30 & 0.025 & 0.034 \\
\hline Melendez and Barbuy (2009) [14] & Fe II & 5 & 0.030 & 0.035 \\
\hline Lawler et al. (2013) [25] & Ti I & 26 & 0.030 & 0.048 \\
\hline Blackwell et al. (1982) rescaled using O'Brian et al. (1991) [26,27] & Fe I & 6 & 0.030 & 0.028 \\
\hline Bridges priv. comm. with NIST (1976) [5] & Cr I & 12 & 0.040 & 0.036 \\
\hline Blackwell et al. (1982) [26] & Fe I & 10 & 0.040 & 0.037 \\
\hline Bard and Kock (1994) [28] & Fe I & 12 & 0.045 & 0.039 \\
\hline Blackwell et al. (1984) [29] & CrI & 2 & 0.050 & 0.050 \\
\hline Rescaled values of Lennard et al. (1975) [30] & $\mathrm{Ni} I$ & 3 & 0.050 & 0.087 \\
\hline Kostyk (1981) [16] & Cr I & 6 & 0.050 & 0.016 \\
\hline Smith and O'Neill (1975) [31] & $\mathrm{CaI}$ & 3 & 0.050 & 0.026 \\
\hline Blackwell et al. (1979) [32] & Fe I & 3 & 0.050 & 0.029 \\
\hline Ryabchikova et al. (1999) [33] & Fe II & 3 & 0.050 & 0.049 \\
\hline Martin et al. (1988) [34] & $\mathrm{Cr}$ I/Ti I/Mn I & 22 & 0.055 & 0.070 \\
\hline Den Hartog et al. (2014) [21] & $\mathrm{Fe} I$ & 12 & 0.055 & 0.095 \\
\hline Wood et al. (2014) [35] & $\mathrm{Ni}$ I & 21 & 0.060 & 0.082 \\
\hline Bard and Kock (1994) rescaled using O'Brian et al. $(1991)[27,28]$ & Fe I & 3 & 0.060 & 0.025 \\
\hline Ruffoni et al. (2014) [36] & Fe I & 11 & 0.070 & 0.083 \\
\hline O'Brian et al. (1991) [27] & Fe I & 29 & 0.070 & 0.130 \\
\hline Bard et al. (1991) rescaled using O'Brian et al. $(1991)[20,27]$ & Fe I & 2 & 0.070 & 0.040 \\
\hline Raassen and Uylings (1998a) [37] & Fe II & 7 & 0.070 & 0.049 \\
\hline Wood et al. (2013) [38] & Ti II & 9 & 0.070 & 0.039 \\
\hline Bard et al. (1991) [20] & Fe I & 3 & 0.070 & 0.037 \\
\hline Pickering et al. (2002) [39] & Ti II & 8 & 0.075 & 0.129 \\
\hline Hannaford et al. (1982) [40] & Y II & 2 & 0.075 & 0.045 \\
\hline Wickliffe and Lawler (1997) [41] & $\mathrm{Ni}$ I & 3 & 0.080 & 0.038 \\
\hline Blackwell et al. (1986) [23] & Ti I & 12 & 0.080 & 0.037 \\
\hline May et al. (1974) [42] & Fe I & 82 & 0.080 & 0.096 \\
\hline Kostyk and Orlova (1983) [43] & Cr II/Ti II & 3 & 0.090 & 0.074 \\
\hline Blackwell et al. (1980) [44] & Fe II & 9 & 0.100 & 0.131 \\
\hline Kurucz and Bell (1995) [45] & Si I & 7 & 0.100 & 0.033 \\
\hline Garz (1973) [10] & Si I & 7 & 0.100 & 0.033 \\
\hline Wiese and Martin (1990) [46] & * & 25 & 0.100 & 0.243 \\
\hline Fuhr et al. (1988) [47] & $\mathrm{Fe} I / \mathrm{Ni} \mathrm{I}$ & 112 & 0.100 & 0.116 \\
\hline Raassen and Uylings (1998b) [48] & $\mathrm{Cr}$ II/Fe II & 6 & 0.105 & 0.059 \\
\hline NIST (online values before 2004) [5] & $*$ & 63 & 0.110 & 0.119 \\
\hline Blackwell et al. (1982) [49] & Ti I & 2 & 0.110 & 0.020 \\
\hline Kurucz (1999-2014) [50] & * & 153 & 0.120 & 0.229 \\
\hline Schnabel et al. (2004) [51] & Fe II & 4 & 0.125 & 0.183 \\
\hline Lobel (2011) [52] & $*$ & 212 & 0.130 & 0.212 \\
\hline Blackwell et al. (1983) [53] & Ti I & 3 & 0.140 & 0.017 \\
\hline Biémont et al. (2011) [54] & Y II & 2 & 0.140 & 0.010 \\
\hline Lennard et al. (1975) [30] & $\mathrm{Ni} I$ & 2 & 0.145 & 0.145 \\
\hline Doerr and Kock (1985) [55] & $\mathrm{Ni} I$ & 9 & 0.160 & 0.141 \\
\hline Fuhr and Wiese (1998) [56] & $\mathrm{Ca} \mathrm{I}$ & 3 & 0.190 & 0.179 \\
\hline Laverick et al. (2018a) [2] & $*$ & 64 & 0.340 & 1.232 \\
\hline
\end{tabular}

\section{Summary and Future Plans}

In this paper, we summarise the status of the BRASS project to-date and we discuss a number of data-products already available to the astronomy and atomic physics communities. The cross-matched atomic literature, compiled in Paper 1, is available to query and view online at brass.sdf.org, with high-quality benchmark spectra and quality assessment results of Paper 2 are now available to download via the BRASS website. BRASS is currently developing an interactive online interface that will allow users to view stellar spectra alongside atomic data, of which a preliminary version can already be found online. To help users understand, interpret, and exploit the quality assessment results of Paper 2, we have developed a set of 1099 dedicated webpages for the spectral lines investigated. 
We hope that by providing such information both astronomers and atomic physicists alike can fully comprehend and make use of the project results.

We also present a very brief literature comparison with the Paper 2 results to infer possible data production methods that may lead to improved atomic data in the future, and to stimulate further, more in-depth investigations by the atomic community. We summarise our findings and note that the combination of reliable relative line strengths and accurate absolute scaling seems to produce consistent results with the Paper 2 stellar derivations. The brief comparison presented here does not attempt to take into account uncertainties associated with the literature atomic data, but we do acknowledge the substantial efforts put into producing such values, especially for recent theoretical calculations (for a review on theoretical uncertainties, see Chung et al. 2016 [57]). To truly exploit the results of the project, and to use the quality assessment results to benchmark both experimental and theoretical methodologies, one should compare entire datasets against each other. Unfortunately, due to the compilation nature of the line lists and repositories such as VALD and NIST, the cross-matched atomic data do not contain multiple complete sources of atomic data. We hope that further, more in-depth investigations by the atomic data community can provide beneficial and valuable feedback on how to further improve atomic data.

In the coming year, BRASS aims to extend the work to cover a wider range of spectral types and atomic lines, and to investigate potential NLTE effects in hotter A- and B-type stars. A significant number of high-quality, science-ready, normalised stellar spectra will also be made publicly available through a new online interactive spectral database coupled with atomic data. Development will continue on the BRASS website and we aim for a full, working release by the end of 2019.

Author Contributions: Conceptualization, A.L., P.R. and C.M.; Methodology, M.L, A.L., and P.R.; Software, A.L. and E.T.; Investigation, M.L, A.L, P.R., T.M., P.A.M.v.H. and M.V.d.S.; Writing, Review and Editing, M.L., A.L., P.R., M.D. and H.H.; Supervision, A.L, P.R., M.D. and H.H.

Funding: This research was funded by the Federaal Wetenschapsbeleid (BELSPO) under contract No. BR/143/A2/BRASS.

Acknowledgments: We thank the atomic data producers and providers for their invaluable work towards improving the accuracy of stellar spectroscopy and the ease with which such vast quantities of data can be retrieved. The research for the present results has been subsidised by the Belgian Federal Science policy Office under contract No. BR/143/A2/BRASS. T.M. and M.V.d.S are supported by a grant from the Fondation ULB. This work has made use of the VALD database, operated at Uppsala University, the Institute of Astronomy RAS in Moscow, and the University of Vienna. This work was based on observations made with the Mercator Telescope, operated on the island of La Palma by the Flemish Community, at the Spanish Observatorio del Roque de los Muchachos of the Instituto de Astrofísica de Canarias. This work was also based on observations obtained with the HERMES spectrograph, which is supported by the Research Foundation-Flanders (FWO), Belgium; the Research Council of KU Leuven, Belgium; the Fonds National de la Recherche Scientifique (F.R.S.-FNRS), Belgium; the Royal Observatory of Belgium; the Observatoire de Gèneve, Switzerland; and the Thüringer Landessternwarte Tautenburg, Germany.

Conflicts of Interest: The authors declare no conflict of interest.

\section{References}

1. Lobel, A.; Royer, P.; Martayan, C.; Laverick, M.; Merle, T.; David, M.; Hensberge, H.; Thienpont, E. The Belgian repository of fundamental atomic data and stellar spectra. Can. J. Phys. 2017, 95, 833-839. [CrossRef]

2. Laverick, M.; Lobel, A.; Merle, T.; Royer, P.; Martayan, C.; David, M.; Hensberge, H.; Thienpont, E. The Belgian repository of fundamental atomic data and stellar spectra (BRASS)-I. Cross-matching atomic databases of astrophysical interest. Astron. Astrophys. 2018, 612, A60. [CrossRef]

3. Laverick, M.; Lobel, A.; Royer, P.; Martayan, C.; Merle, T.; van Hoof, P.A.M.; van der Swaelmen, M.; David, M.; Hensberge, H.; Thienpont, E. The Belgian repository of fundamental atomic data and stellar spectra (BRASS)-II. Quality assessment of atomic line data for G-type stars. Astron. Astrophys. 2018, submitted. [CrossRef]

4. Ryabchikova, T.; Piskunov, N.; Kurucz, R.L.; Stempels, H.C.; Heiter, U.; Pakhomov, Y.; Barklem, P.S. A major upgrade of the VALD database. Phys. Scripta 2015, 90, 054005. [CrossRef] 
5. $\quad$ Kramida, A.; Ralchenko, Y.; Reader, J.; NIST ASD Team. NIST Atomic Spectra Database (Version 5.5.6). 2018. Available Online: http:/ / physics.nist.gov/asd (accessed on 6 April 2018).

6. Dubernet, M.L.; Antony, B.K.; Ba, Y.A.; Babikov, Y.L.; Bartschat, K.; Boudon, V.; Braams, B.J.; Chung, H.K.; Daniel, F.; Delahaye, F.; et al. The virtual atomic and molecular data centre (VAMDC) consortium. J. Phys. B Atom. Mol. Opt. Phys. 2016, 49, 074003. [CrossRef]

7. Raskin, G.; Van Winckel, H.; Hensberge, H.; Jorissen, A.; Lehmann, H.; Waelkens, C.; Avila, G.; De Cuyper, J.-P.; Degroote, P.; Dubosson, R.; et al. HERMES: A high-resolution fibre-fed spectrograph for the Mercator telescope. Astron. Astrophys. 2011, 526, A69. [CrossRef]

8. Bagnulo, S.; Jehin, E.; Ledoux, C.; Cabanac, R.; Melo, C.; Gilmozzi, R. The ESO Paranal Science Operations Team. Messenger 2003, 114, 10.

9. Neckel, H.; Labs, D. The solar radiation between 3300 and 12500 Å. Solar Phys. 1984, 90, 205-258. [CrossRef]

10. Garz, T. Absolute Oscillator Strengths of SI I Lines between 2500 A and 9000 A. Astron. Astrophys. 1973, 26,471 .

11. O'Brian, T.R.; Lawler, J.E. Radiative lifetimes in Si i from laser-induced fluorescence in the visible, ultraviolet, and vacuum ultraviolet. Phys. Rev. 1991, 44, 7134. [CrossRef]

12. Asplund, M.; Grevesse, N.; Sauval, A.J.; Scott, P. The chemical composition of the Sun. Ann. Rev. Astron. 2009, 47, 481-522. [CrossRef]

13. Scott, P.; Grevesse, N.; Asplund, M.; Bergemann, M.; Sauval, A.J. The elemental composition of the Sun-II. The iron group elements Sc to Ni. Astron. Astrophys. 2015, 573, A26. [CrossRef]

14. Melendez, J., Barbuy, B. Both accurate and precise gf-values for Fe II lines. Astron. Astrophys. 2009, 497, 611-617. [CrossRef]

15. Hinkle, K.; Wallace, L.; Valenti, J.; Harmer, D. Visible and Near Infrared Atlas of the Arcturus Spectrum 3727-9300 A; Hinkle, K., Wallace, L., Valenti, J., Harmer, D., Eds.; ASP: San Francisco, CA, USA, 2000; Volume 2, ISBN 1-58381-037-4.

16. Kostyk, R.I. Oscillator strengths for lines of neutral chromium. Astrom. Astrofiz. 1981, 45, 3-9. (In Russian)

17. Kostyk, R.I. Oscillator Strengths for Neutral Titanium Lines. Sov. Astron. 1982, 26, 422-426.

18. Kostyk, R.I. Oscillator strengths of lines of neutral nickel. Astrom. Astrofiz. 1982, 46, 58.

19. Pehlivan Rhodin, A.; Hartman, H.; Nilsson, H.; Jönsson, P. Experimental and theoretical oscillator strengths of Mg I for accurate abundance analysis. Astron. Astrophys. 2017, 598, A102. [CrossRef]

20. Bard, A.; Kock, A.; Kock, M. Fe I oscillator strengths of lines of astrophysical interest. Astron. Astrophys. 1991, 248, 315-322.

21. Den Hartog, E.A.; Ruffoni, M.P.; Lawler, J.E.; Pickering, J.C.; Lind, K.; Brewer, N.R. Fe I Oscillator Strengths for Transitions from High-lying Even-parity Levels. Astrophys. J. Suppl. 2014, 215, 23. [CrossRef]

22. Sobeck, J.S.; Lawler, J.S.; Sneden, C. Improved laboratory transition probabilities for neutral chromium and redetermination of the chromium abundance for the sun and three stars. Astrophys. J. 2007, 667, 1267. [CrossRef]

23. Blackwell, D.E.; Booth, A.J.; Menon, S.L.R.; Petford, A.D. Measurement of relative oscillator strengths for Ti I-IV. Transitions from levels of excitation energy between 1.42 and $2.31 \mathrm{eV}$. Mon. Not. Roy. Astron. Soc. 1986, 220, 289-302. [CrossRef]

24. Grevesse, N.; Blackwell, D.E.; Petford, A.D. Revision of the absolute scale of the Oxford Ti i oscillator strengths and the solar titanium abundance. Astron. Astrophys. 1989, 208, 157.

25. Lawler, J.E.; Guzman, A.; Wood, M.P.; Sneden, C.; Cowan, J.J. Improved Log (gf) Values for Lines of Ti I and Abundance Determinations in the Photospheres of the Sun and Metal-Poor Star HD 84937 (Accurate Transition Probabilities for Ti I). Astrophys. J. Suppl. 2013, 205, 11. [CrossRef]

26. Blackwell, D.E.; Petford, A.D.; Shallis, M.J.; Simmons, G.J. Precision measurement of relative oscillator strengths-IX. Measures of Fe i transitions from levels. Mon. Not. Roy. Astron. Soc. 1982, 199, 43-51. [CrossRef]

27. O’Brian, T.R.; Wickliffe, M.E.; Lawler, J.E.; Whaling, W.; Brault, J.W. Lifetimes, transition probabilities, and level energies in Fe I. J. Opt. Soc. Am. B Opt. Phys. 1991, 8, 1185-1201. [CrossRef]

28. Bard, A.; Kock, M. Fe I oscillator strengths for lines with excitation energies between 3 and $7 \mathrm{eV}$. Astron. Astrophys. 1994, 282, 1014-1020. 
29. Blackwell, D.E.; Menon, S.L.R.; Petford, A.D. Measurement of relative oscillator strengths for Cr I lines-I. Measures for transitions from levels $a^{7} S_{3}(0.00 \mathrm{eV}), a^{5} S_{2}(0.94 \mathrm{eV})$ and $a^{5} D_{0,4}(0.96-1.03 \mathrm{eV})$. Mon. Not. R. Astron. Soc. 1984, 207, 533-546. [CrossRef]

30. Lennard, W.N.; Whaling, W.; Scalo, J.M.; Testerman, L. Ni I transition probabilities and the solar nickel abundance. Astrophys. J. 1975, 197, 517-526. [CrossRef]

31. Smith, G.; O'Neill, J.A. Absolute transition probabilities for some lines of neutral calcium. Astron. Astrophys. 1975, 38, 1-4.

32. Blackwell, D.E.; Petford, A.D.; Shallis, M.J. Precision measurement of relative oscillator strengths.-VVI. Measures of Fe I transitions from levels $a^{5} F_{1,5}(0.86-1.01 \mathrm{eV})$ with an accuracy of 0.5 per cent. Mon. Not. R. Astron. Soc. 1979, 186, 657-668. [CrossRef]

33. Ryabchikova, T.A.; Piskunov, N.E.; Stempels, H.C.; Kupka, F.; Weiss, W.W. The Vienna Atomic Line Data Base-A Status Report. Phys. Scr. 1999, T83, 162-173. [CrossRef]

34. Martin, G.; Fuhr, J.; Wiese, W. Atomic Transition Probabilities Scandium through Manganese. J. Phys. Chem. Ref. Data Suppl. 1988, 17, 3.

35. Wood, M.P.; Lawler, J.E.; Sneden, C.; Cowan, J.J. Improved Ni I log (gf) Values and Abundance Determinations in the Photospheres of the Sun and Metal-poor Star HD 84937. Astrophys. J. Suppl. 2014, 211, 20. [CrossRef]

36. Ruffoni, M.P.; Den Hartog, E.A.; Lawler, J.E.; Brewer, N.R.; Lind, K.; Nave, G.; Pickering, J.C. Fe I oscillator strengths for the Gaia-ESO survey. Mon. Not. R. Astron. Soc. 2014, 441, 3127-3136. [CrossRef]

37. Raassen, A.; Uylings, P. Critical evaluation of calculated and experimental transition probabilities and lifetimes for singly ionized iron group elements. J. Phys. B 1998, 31, 3137-3146. [CrossRef]

38. Wood, M.P.; Lawler, J.E.; Sneden, C.; Cowan, J.J. Improved Ti II log (gf) values and abundance determinations in the photospheres of the sun and metal-poor star HD 84937. Astrophys. J. Suppl. 2013, $208,27$.

39. Pickering, J.C.; Thorne, A.P.; Perez, R. Oscillator Strengths of Transitions in Ti II in the Visible and Ultraviolet regions. Astrophys. J. Suppl. 2001, 132, 403; Erratum in 2002, 138, 247-248. [CrossRef]

40. Hannaford, F.; Lowe, R.M.; Grevesse, N.; Biemont, E.; Whaling, W. Oscillator strengths for YI and Y II and the solar abundance of yttrium. Astrophys. J. 1982, 261, 736-746. [CrossRef]

41. Wickliffe, M.E.; Lawler, J.E. Atomic Transition Probabilities in Ni I. Astrophys. J. Suppl. 1997, $110,163$. [CrossRef]

42. May, M.; Richter, J.; Wichelmann, J. Experimental oscillator strengths of weak FeI lines. Astron. Astrophys. 1974, 18, 405.

43. Kostyk, R.I.; Orlova, T.V. Oscillator strengths of lines of ionized titanium and chromium. Astrom. Astrofiz. 1983, 49, 39-41. (In Russian)

44. Blackwell, D.E.; Shallis, M.J.; Simmons, G.J. Oscillator strengths of Fe II lines derived from the solar spectrum-Choice of solar model atmosphere. Astron. Astrophys. 1980, 81, 340-343.

45. Kurucz, R.L.; Bell, B. Kurucz CD-Rom No. 23. Harvard-Smithsonian Center for Astrophysics: Cambridge, MA, USA, 1995.

46. Wiese W.L.; Martin G.A. Wavelengths and Transition Probabilities for Atoms and Atomic Ions. Available online: http:/ / adsabs.harvard.edu/abs/1980wtpa.book.....W (accessed on 6 April 2018).

47. Fuhr, J.R.; Martin, G.A.; Wiese, W.L. Atomic transition probabilities. Iron through Nickel. J. Phys. Chem. Ref. Data Suppl. 1988, 17, 4 .

48. Raassen, A.; Uylings, P. On the determination of the solar iron abundance using Fe II lines. Astron. Astrophys. 1998, 340, 300-304.

49. Blackwell, D.E.; Menon, S.L.R.; Petford, A.D.; Shallis, M.J. Precision measurement of relative oscillator strengths for Ti I-II. Transitions from levels (1.5-1.07 eV). Mon. Not. R. Astron. Soc. 1982, 201, 611-617. [CrossRef]

50. Kurucz, R.L.; Robert, L. Kurucz On-Line Database of Observed and Predicted Atomic Transitions. Available online: http:/ / kurucz.harvard.edu/atoms/ (accessed on 6 April 2018).

51. Schnabel, R.; Schultz-Johanning, M.; Kock, M. Fe II lifetimes and transition probabilities. Astron. Astrophys. 2004, 414, 1169-1176. [CrossRef]

52. Lobel, A. Oscillator strength measurements of atomic absorption lines from stellar spectra. Can. J. Phys. 2011, 89, 395-402. [CrossRef] 
53. Blackwell, D.E.; Menon, S.L.R.; Petford, A.D. Measurement of relative oscillator strengths for Ti I-III. Weak transitions from levels $a^{3} F_{3,4}(0.02 \mathrm{eV}, 0.05 \mathrm{eV}), a^{5} F_{1,5}(0.81 \mathrm{eV}-0.85 \mathrm{eV}), a^{1} D_{2}(0.90 \mathrm{eV}), a^{3} P_{0,3}(1.05 \mathrm{eV}-1.07 \mathrm{eV})$ with solar analysis. Mon. Not. R. Astron. Soc. 1983, 204, 883-890. [CrossRef]

54. Biémont, E.; Blagoev, K.; Engstrom, L.; Hartman, H.; Lundberg, H.; Malcheva, G.; Nilsson, H.; Whitehead, R.B.; Palmeri, P.; Quinet, P. Lifetime measurements and calculations in $\mathrm{Y}^{+}$and $\mathrm{Y}^{2+}$ ions. Mon. Not. R. Astron. Soc. 2011, 414, 3350-3359. [CrossRef]

55. Doerr, A.; Kock, M. Ni I oscillator strengths. J. Quant. Spectrosc. Radiat. Transf. 1985, 33, 307-318. [CrossRef]

56. Fuhr, J.R.; Wiese, W.L. CRC Handbook of Chemistry and Physics, 79th ed.; 10-88-10-146; Lide, D.R., Ed.; CRC Press: Boca Raton, FL, USA, 1998.

57. Chung, H.-K.; Braams, B.J.; Bartschat, K.; Császár, A.G.; Drake, G.W.F.; Kirchner, Y.; Kokoouline, V.; Tennyson, J. Uncertainty estimates for theoretical atomic and molecular data. J. Phys. D Appl. Phys. 2016, 36, 363002.

(C) 2018 by the authors. Licensee MDPI, Basel, Switzerland. This article is an open access article distributed under the terms and conditions of the Creative Commons Attribution (CC BY) license (http://creativecommons.org/licenses/by/4.0/). 